\title{
A primeira vez a gente não esquece: conhecendo as drogas experimentadas por estudantes do ensino médio
}

\author{
Eliany Nazaré Oliveira ${ }^{1}$ \\ (D) https://orcid.org/0000-0002-6408-7243 \\ Joyce Mazza Nunes ${ }^{1,2}$ \\ (D) https://orcid.org/0000-0003-2865-579x \\ Maristela Inês Osawa Vasconcelos \\ (D) https://orcid.org/0000-0002-1937-8850 \\ Lorenna Saraiva Viana ${ }^{2,3}$ \\ (D) https://orcid.org/0000-0003-1496-5164 \\ Roberta Magda Martins Moreira 2,3 \\ (D) https://orcid.org/0000-0002-8225-7576 \\ Mariana Rodrigues Bezerra ${ }^{1}$ \\ (D) https://orcid.org/0000-0003-3785-5558
}

* Apoio financeiro do Conselho Nacional de Desenvolvimento Científico e Tecnológico, Brasil, n 21/2018

${ }^{1}$ Universidade Estadual Vale do Acaraú, Campus Sobral, Sobral, CE, Brasil.

2 Universidade Federal do Ceará, Campus Sobral, Sobral, CE, Brasil.

${ }^{3}$ Scholarship holder at the Coordenação de Aperfeiçoamento de Pessoal de Nível Superior (CAPES), Brasil.

\begin{abstract}
Objetivo: investigar a prevalência do consumo de drogas em escolas de ensino médio de um município referência do estado do Ceará. Método: estudo exploratório descritivo com abordagem quantitativa. A coleta de dados foi realizada no período de março a julho de 2019 com 562 estudantes. Para tanto, utilizou-se o questionário para triagem do uso de álcool, tabaco e outras substâncias. Na análise, realizouse estatística analítica por meio do teste de qui-quadrado. Resultados: em relação as substâncias já experimentadas pelos estudantes, o álcool aparece em maior proporção. Além do álcool, os estudantes também já experimentaram outras substâncias psicoativas, em que maconha, tabaco e hipnóticos ou sedativos tiveram destaque entre as já experimentadas. Em relação aos parâmetros, para todas as substâncias, houve uma proporção variando entre $(1,5 \%$ a $23,2 \%$ ) de estudantes necessitando de intervenção breve. Conclusão: estes resultados apresentados contribuem para uma melhor compreensão do fenômeno no que diz respeito às substâncias mais consumidas e padrões de uso nocivo ou dependência, para que possa planejar e implementar programas de prevenção primária e secundária no contexto escolar.
\end{abstract}

Descritores: Drogas; Instituições Acadêmicas; Adolescente; Estudantes.

\section{Como citar este artigo}

Oliveira EN, Nunes JM, Vasconcelos MIO, Viana LS, Moreira RMM, Bezerra MR. The first time we don't forget: Knowing the drugs experienced by high school students. SMAD, Rev Eletrônica Saúde Mental Álcool Drog. 2020;16(2):1-8. doi: https://dx.doi.org/10.11606/issn.1806-6976.smad.2020.165488 


\title{
The first time we don't forget: Knowing the drugs experienced by high school students
}

\begin{abstract}
Objective: this study aims to investigate the prevalence of drug use in two secondary schools in a reference municipality of the state of Ceará. Method: a descriptive and exploratory study with a quantitative approach. Data collection occurred from March to July 2019, with 562 students. For this purpose, we used the questionnaire for screening the use of alcohol, tobacco, and other substances. Results: regarding the substances the students had already tried, alcohol appears in greater proportion. In addition to alcohol, the students had also tried other psychoactive substances such as marijuana, tobacco, and hypnotics or sedatives, which all got emphasis among the substances already tried by the students. Regarding the parameters for all substances, there was a proportion ranging from $1.5 \%$ to $23.2 \%$ of students presenting need for brief interventions. Conclusion: the results presented contribute to a better understanding of the phenomenon regarding the most consumed substances, and of the harmful use or addiction patterns, so that we can plan and implement primary and secondary prevention programs inside the school context.
\end{abstract}

Descriptors: Drugs; Schools; Adolescent; Students.

\section{La primera vez no se olvida: conociendo las drogas que consumen los estudiantes de secundaria}

Objetivo: investigar la prevalencia del consumo de drogas en escuelas secundarias de un municipio referencia del estado de Ceará. Método: estudio exploratorio y descriptivo con enfoque cuantitativo. La recolección de datos se realizó en el periodo de marzo a julio de 2019 con 562 estudiantes. Para ello se utilizó el cuestionario para clasificación del consumo de alcohol, tabaco y otras sustancias. En el análisis se realizó estadística analítica por medio del test de chicuadrado. Resultados: en relación a las sustancias ya probadas por los estudiantes, el alcohol aparece en mayor proporción. Además del alcohol, los estudiantes ya probaron otras sustancias psicoactivas, destacándose la marihuana, el tabaco y los hipnóticos o sedantes. En relación a los parámetros para todas las sustancias, se registró una proporción que varió entre 1,5\% y 23,2\% de estudiantes con necesidad de intervenciones rápidas. Conclusión: los resultados presentados ayudan a comprender mejor el fenómeno en relación a las sustancias más consumidas y a los patrones de uso nocivo o de dependencia, para poder planificar e implementar programas de prevención primaria y secundaria en el contexto escolar.

Descriptores: Drogas; Instituciones Académicas; Adolescent; Estudiantes. 


\section{Introdução}

A droga e seu consumo é um fenômeno antigo e envolve questões culturais, religiosas, econômicas, políticas e sociais. Em várias culturas, sociedades e épocas, os homens consumiram drogas lícitas ou ilícitas. O uso de drogas é um fenômeno social universal, presente em todas as épocas e culturas, variando apenas o status que a droga adquiriu ao longo do tempo e em cada sociedade, ao ter sua produção e distribuição regulamentadas ou, ao contrário, relegadas à informalidade e ilegalidade, na tentativa invariavelmente fracassada de que não sejam consumidas(1).

O Relatório Mundial Sobre Drogas da Organização das Nações Unidas aponta que cerca de 246 milhões de pessoas, com idade entre 15 e 64 anos, tenham usado alguma droga ilícita pelo menos uma vez em 2013 e aproximadamente 27 milhões de pessoas são usuários de drogas problemáticos. Entre os jovens, as chances de vivenciar tais problemas aumentam, uma vez que evidencia uma exposição cada vez mais precoce às substâncias(2).

Uma pesquisa com objetivo de avaliar a relação entre uso de drogas e comportamento antissocial com 7.176 adolescentes de escolas públicas no Brasil, apontou que mais de $80 \%$ dos adolescentes que fizeram uso de bebidas alcoólicas e cigarro tinham entre 14 e $17 \operatorname{anos}^{(3)}$. O uso na vida de drogas ilícitas é quatro vezes mais prevalente nos estudantes que relataram o binge drinking, que consiste no uso excessivo de álcool, com cinco ou mais bebidas alcoólicas no mesmo momento ou dia(4).

A adolescência é um momento significativo na vida do indivíduo. Neste período, o jovem rejeita orientações, pois está testando a possibilidade de ser adulto, de ter poder e controle sobre si mesmo(5). É um momento de diferenciação em que "naturalmente" se afasta da família e adere ao seu grupo de iguais. Se esse grupo estiver experimentalmente usando drogas, o pressiona a usar também. $E$ ao entrar em contato com drogas nesse período de maior vulnerabilidade, expõe-se a inúmeros riscos(5).

$O$ adolescente que se encontra na condição de vulnerabilidade e risco, poderá buscar o alívio e/ou proteção relacionados à situação de estresse, ansiedade ou angústia vivenciadas nesta fase, com a utilização de substâncias psicoativas, podendo desenvolver uma relação de dependência ou um uso abusivo(6).

Em estudos com adolescentes do ensino médio, os resultados indicaram que as substâncias lícitas como álcool e cigarro foram as que apresentaram maior consumo, e o uso de anfetaminas e ansiolíticos tem aumentado nessa população. Isso merece atenção, pelo fato de aquelas serem substâncias lícitas e de estas terem um consumo cujas consequências não são tão visíveis para a sociedade ${ }^{(7)}$.
Pesquisa com 60.973 estudantes de escolas púbicas e privadas das capitais brasileiras e do distrito federal, revelou a gravidade do problema do álcool e drogas entre os adolescentes escolares, evidenciando a precocidade da exposição e a magnitude do problema, apontando que mais de $70 \%$ já foram expostos ao álcool e cerca de $8 \%$ às drogas ilícitas( ${ }^{(8)}$.

Um estudo realizado em um Centro de Atenção Psicossocial Álcool e outras drogas (CAPS AD) evidenciou que a maioria dos usuários em tratamento iniciaram o consumo da substância psicoativa/problema na adolescência(9), em que diversos outros estudos trazem esta faixa etária como a responsável pelo início do consumo(10-12).

O uso e abuso de drogas deve ser uma das principais preocupações da sociedade e o espaço escolar tem sido considerado um lugar privilegiado para o desenvolvimento da prevenção(13). Neste cenário, a escola é um dos espaços mais propícios para o fortalecimento de comportamentos saudáveis e resistência ao uso de drogas.

Alguns autores afirmam que a prevenção ao uso de drogas em escolas é uma estratégia utilizada em todo o mundo, pois visam à promoção da saúde entre os jovens. A escola é um dos locais onde estes jovens permanecem vinculados por um período de tempo considerável, com trocas e aprendizagens significativas ${ }^{(14)}$.

Após esta contextualização, tem-se como questões norteadoras: Quais as principais drogas que os estudantes das escolas analisadas já experimentaram na vida? Qual a prevalência das categorias de severidade de uso de substâncias definidas pelo ASSIST nas escolas? Neste contexto, o objetivo deste estudo é investigar a prevalência do consumo de drogas em escolas de ensino médio de um município referência do estado do Ceará.

\section{Método}

Trata-se de um estudo exploratório descritivo com abordagem quantitativa, realizado em duas escolas de ensino médio de um município referência do estado do Ceará, Sobral - Ceará, o qual foi classificado com nível alto relacionado a problemas graves com a droga ${ }^{(15)}$. Para proteção de suas identidades, as escolas foram denominadas $(X)$ e $(Y)$.

A coleta de dados foi realizada no período de março a julho de 2019, com 562 estudantes do ensino médio, sendo 259 da escola (X) e 305 da escola (Y), em que para delimitá-la foi feito cálculo da variável desfecho qualitativa supondo população finita, uma vez que na escola $X$ apresentava 380 alunos e na $Y, 470$ alunos, para isso, fixou-se um nível de significância de $99 \%$, proporção de $50 \%$ e erro absoluto de $5 \%$, totalizando 242 para a escola X e 276 para a Y. Destaca-se que foi coletado cerca $7 \%$ a mais do que o proposto, a fim de diminuir o viés de perda amostral. 
Os alunos foram selecionados sob o critério de estarem presentes no momento da coleta com consentimentos dos pais para participar da pesquisa e foram excluídos aqueles que apresentaram algum déficit cognitivo ou que dificultasse a comunicação e o preenchimento completo do questionário, os quais participaram, mas foram excluídos posteriormente, ao ser percebida essa limitação na comunicação e nas múltiplas variáveis não preenchidas.

Para tanto, utilizou-se o questionário para triagem do uso de álcool, tabaco e outras substâncias (ASSIST - OMS Vs3.1) validado para o Brasil(16), este já é utilizado em várias pesquisas no território nacional. É um questionário estruturado que contém oito questões sobre o uso de nove classes de substâncias psicoativas (tabaco, álcool, maconha, cocaína, estimulantes, sedativos, inalantes, alucinógenos, e opiáceos). As perguntas abordam a frequência de uso, na vida e nos últimos três meses, problemas relacionados ao uso, preocupação a respeito do uso por parte de pessoas próximas ao usuário, prejuízo na execução de tarefas esperadas, tentativas malsucedidas de cessar ou reduzir o consumo, sentimento de compulsão e uso por via injetável(17).

No ASSIST, os escores das respostas variam de 0 a 33 pontos, tendo sido usado o valor do escore total para cada uma das sustâncias psicoativas para a realização dos testes estatísticos. Entretanto, assinalam-se os níveis que vão desde uso ocasional, abuso, até dependência. Considera-se a faixa de 0 a 3 como indicativos de uso ocasional, escores de 4 a 26 como indicativo de abuso, e de 27 ou mais como sugestivo de dependência. Cabe ressaltar que a pontuação para avaliar o padrão de consumo de álcool é diferenciada das demais substâncias. Para o álcool, considera-se uma tolerância maior com escores entre 0 a 10 como indicativo de uso ocasional; de 11 a 26 como indicativo de abuso; e de escores de 27 ou mais, sugerindo dependência(17-18).

A análise da associação entre as duas escolas e as características sociodemográficas, substância utilizada e o método de intervenção foi feita pelos testes de Quiquadrado e de razão de verossimilhança. Para essas análises, foram consideradas como estatisticamente significantes as com $\mathrm{p}<0,05$. Os dados foram processados no SPSS 20,0, licença número 10101131007.

O estudo foi orientado pela Resolução 466/12(19), que estabelece diretrizes para este procedimento com seres humanos, tendo sido aprovado com o Parecer $n^{\circ} 2.989 .395$.

\section{Resultados}

O consumo de drogas é apontado como uma das principais preocupações da sociedade e a escola tem sido considerada um espaço privilegiado para o desenvolvimento da prevenção, para isso, torna-se necessário conhecer o perfil dos estudantes, para intervir conforme as necessidades desse público. Logo, avaliou-se os dados sociodemográfico dos estudantes, apresentados na Tabela 1.

Tabela 1 - Distribuição do número de estudantes, segundo as escolas e características como sexo, idade (anos), ano/série, estado civil e religião, Sobral, Ceará, Brasil, 2019

\begin{tabular}{lccccc}
\hline \multirow{2}{*}{ Característica } & \multicolumn{2}{c}{ Escola $\mathbf{X}$} & \multicolumn{2}{c}{ Escola $\mathbf{Y}$} & \multirow{2}{*}{$\mathbf{P}^{*}$} \\
\cline { 2 - 5 } & $\mathbf{n}$ & $\%$ & $\mathbf{N}$ & $\%$ & \\
\hline Sexo & 121 & 46,7 & 175 & 57,4 & \\
$\quad$ Feminino & 138 & 53,3 & 130 & 42,6 & \\
$\quad$ Masculino & & & & & $\mathbf{0 , 0 0 6}$ \\
Idade (anos) & 43 & 16,6 & 32 & 10,6 & \\
14 & 96 & 37,1 & 87 & 28,7 & \\
15 & 62 & 23,9 & 101 & 33,3 & \\
16 & 58 & 22,4 & 83 & 27,4 & \\
17 & & & & & $\mathbf{0 , 0 0 0}$ \\
Ano/ série & 112 & 43,2 & 90 & 29,4 & \\
$1^{10}$ ano & 89 & 34,4 & 81 & 26,5 & \\
$2^{\circ}$ ano & 58 & 22,4 & 135 & 44,1 & \\
$3^{\circ}$ ano & & & & & $\mathbf{0 , 0 0 1}$ \\
Estado civil & 238 & 91,9 & 299 & 97,7 & \\
Solteiro & 21 & 8,1 & 7 & 2,3 & \\
Casado/ união estável & & & & & $\mathbf{0 , 9 5}$ \\
Religião & 223 & 86,1 & 263 & 85,9 & \\
$\quad$ Sim & 36 & 13,9 & 43 & 14,1 & \\
$\quad$ Não & & & & & \\
\hline *p do teste de Qui-Quadrado &
\end{tabular}

*p do teste de Qui-Quadrado

Logo, observou-se que sexo, idade, série e estado civil apresentam diferença estatisticamente significativa ao comparar entre as escolas $(p<0,05)$, em que o sexo masculino $(53,3 \%)$ foi predominante na escola $X$, enquanto que na escola $Y$, a maioria era do sexo feminino $(57,4 \%)$.

Em relação a idade, os adolescentes de 15 anos estão em maior quantidade na escola $X$, com $37,1 \%$, e os de 16 anos aparecem em maior proporção na escola $Y$, com 33,3\%. Os estudantes do $1^{\circ}$ ano compuseram maior número na escola $X$, com 43,2\%, e na escola $Y$, destacou-se os estudantes do $3^{\circ}$ ano $(44,1 \%)$.

Quanto ao estado civil, a maioria dos estudantes de ambas as escolas eram solteiros. No item religião, não houve diferença estatisticamente significativa entre as escolas $X$ e $Y$, em que a maior parte dos alunos informaram possuir religião.

Ao avaliar o uso de drogas nas escolas, obteve-se os seguintes resultados presentes na Tabela 2 .

A Tabela 3, destaca que apenas o uso de álcool, maconha e cocaína/crack apresentaram diferença estatisticamente significante ao comparar as escolas $X$ e $Y$, com $p<0,05$. Quanto ao álcool, 59,8\% já haviam consumido na escola $X$ e $76,1 \%$ na escola $Y$. Em relação a maconha, 28,6\% experimentaram na escola $X$ e $12,7 \%$ na $Y$ e, 5,0\% usaram crack na escola $X$ e 1,3\% na $Y$. 
Tabela 2 - Distribuição do número de estudantes, segundo o uso de drogas nas escolas, Sobral, Ceará, Brasil, 2019

\begin{tabular}{|c|c|c|c|c|c|}
\hline \multirow{2}{*}{ Substância } & \multicolumn{2}{|c|}{ Escola $X$} & \multicolumn{2}{|c|}{ Escola Y } & \multirow[t]{2}{*}{$\mathbf{p}^{*}$} \\
\hline & $\mathrm{n}$ & $\%$ & $\mathbf{N}$ & $\%$ & \\
\hline Tabaco & & & & & 0,88 \\
\hline Sim & 47 & 18,1 & 57 & 18,6 & \\
\hline Não & 212 & 81,9 & 249 & 81,4 & \\
\hline Álcool & & & & & 0,000 \\
\hline Sim & 155 & 59,8 & 233 & 76,1 & \\
\hline Não & 104 & 40,2 & 73 & 23,9 & \\
\hline Maconha & & & & & 0,000 \\
\hline Sim & 74 & 28,6 & 39 & 12,7 & \\
\hline Não & 185 & 71,4 & 267 & 87,3 & \\
\hline Cocaína/Crack & & & & & 0,01 \\
\hline Sim & 13 & 5,0 & 4 & 1,3 & \\
\hline Não & 246 & 95,0 & 302 & 98,7 & \\
\hline Anfetaminas ou êxtase & & & & & 0,26 \\
\hline Sim & 9 & 3,5 & 6 & 2,0 & \\
\hline Não & 250 & 96,5 & 300 & 98,0 & \\
\hline Inalantes & & & & & 0,50 \\
\hline Sim & 38 & 14,7 & 39 & 12,7 & \\
\hline Não & 221 & 85,3 & 267 & 87,3 & \\
\hline Hipnóticos ou sedativos & & & & & 0,30 \\
\hline Sim & 16 & 6,2 & 13 & 4,2 & \\
\hline Não & 243 & 93,8 & 293 & 95,8 & \\
\hline Alucinógenos & & & & & 0,36 \\
\hline Sim & 6 & 2,3 & 4 & 1,3 & \\
\hline Não & 253 & 97,7 & 302 & 98,7 & \\
\hline Opióides ou opiáceos & & & & & 0,53 \\
\hline Sim & 2 & 0,8 & 4 & 1,3 & \\
\hline Não & 257 & 99,2 & 302 & 98,7 & \\
\hline Outras drogas & & & & & 0,12 \\
\hline Sim & 4 & 1,5 & 1 & 0,3 & \\
\hline Não & 255 & 98,5 & 305 & 99,7 & \\
\hline
\end{tabular}

A partir desses dados, investigou-se os métodos de intervenção indicados para os estudantes, definidos pelo ASSIST, conforme as escolas, apresentados na Tabela 3.

Com o objetivo de explorar melhor a severidade no envolvimento com as substâncias entre os participantes, os escores brutos foram codificados de acordo com os pontos de corte recomendados para o ASSIST. Esses pontos de corte descrevem o quanto os estudantes estão consumindo a substância e em que medida este uso já é prejudicial, resultando em três categorias possíveis, em ordem crescente de severidade: a) sem necessidade de intervenção, b) com necessidade de intervenção breve e c) necessidade de intervenção intensiva.

Nesse sentido, observou-se diferença estatisticamente significativa ao comparar as escolas, referentes às intervenções por uso de álcool, maconha, cocaína/crack e inalantes. Verifica-se que o álcool $(1,9 \%)$, tabaco $(0,8 \%)$, maconha $(0,4 \%)$, crack/cocaína $(0,4 \%)$, inalantes $(0,4 \%)$ na escola $X$ necessitam de uma intervenção intensiva. No entanto, em todas as substâncias, existiu uma proporção variando entre $(1,5 \%$ a $23,2 \%)$ acerca da necessidade de intervenção breve.
Tabela 3 - Prevalência dos métodos de intervenção definidos pelo ASSIST, conforme as escolas, Sobral, Ceará, Brasil, 2019

\begin{tabular}{|c|c|c|c|c|c|}
\hline \multirow{2}{*}{ Substância } & \multicolumn{2}{|c|}{ Escola X } & \multicolumn{2}{|c|}{ Escola Y } & \multirow[t]{2}{*}{$\mathbf{p}^{*}$} \\
\hline & $\mathbf{n}$ & $\%$ & $\mathbf{N}$ & $\%$ & \\
\hline Tabaco & & & & & 0,716 \\
\hline Nenhuma Intervenção & 234 & 90,3 & 276 & 91,7 & \\
\hline Intervenção Breve & 23 & 8,9 & 24 & 8,0 & \\
\hline Encaminhamento Intensivo & 2 & 0,8 & 1 & 0,3 & \\
\hline Álcool & & & & & 0,001 \\
\hline Nenhuma Intervenção & 194 & 74,9 & 259 & 85,8 & \\
\hline Intervenção Breve & 60 & 23,2 & 43 & 14,2 & \\
\hline Encaminhamento Intensivo & 5 & 1,9 & 0 & 0,0 & \\
\hline Maconha & & & & & 0,000 \\
\hline Nenhuma Intervenção & 199 & 76,8 & 276 & 91,7 & \\
\hline Intervenção Breve & 59 & 22,8 & 25 & 8,3 & \\
\hline Encaminhamento Intensivo & 1 & 0,4 & 0 & 0,0 & \\
\hline Cocaína/ Crack & & & & & 0,027 \\
\hline Nenhuma Intervenção & 246 & 95,0 & 297 & 98,7 & \\
\hline Intervenção Breve & 12 & 4,6 & 4 & 1,3 & \\
\hline Encaminhamento Intensivo & 1 & 0,4 & 0 & 0,0 & \\
\hline Anfetaminas ou Exxtase & & & & & 0,114 \\
\hline Nenhuma Intervenção & 252 & 97,3 & 299 & 99,3 & \\
\hline Intervenção Breve & 6 & 2,3 & 2 & 0,7 & \\
\hline Encaminhamento Intensivo & 1 & 0,4 & 0 & 0,0 & \\
\hline Inalantes & & & & & 0,034 \\
\hline Nenhuma Intervenção & 237 & 91,5 & 290 & 96,3 & \\
\hline Intervenção Breve & 21 & 8,1 & 11 & 3,7 & \\
\hline Encaminhamento Intensivo & 1 & 0,4 & 0 & 0,0 & \\
\hline Hipnóticos ou Sedativos & & & & & 0,064 \\
\hline Nenhuma Intervenção & 245 & 94,6 & 295 & 98,0 & \\
\hline Intervenção Breve & 13 & 5,0 & 6 & 2,0 & \\
\hline Encaminhamento Intensivo & 1 & 0,4 & 0 & 0,0 & \\
\hline Alucinógenos & & & & & 0,397 \\
\hline Nenhuma Intervenção & 253 & 97,7 & 295 & 98,7 & \\
\hline Intervenção Breve & 5 & 1,9 & 4 & 1,3 & \\
\hline Encaminhamento Intensivo & 1 & 0,4 & 0 & 0,0 & \\
\hline Opióides ou opiáceos & & & & & 0,391 \\
\hline Nenhuma Intervenção & 254 & 98,1 & 297 & 99,0 & \\
\hline Intervenção Breve & 4 & 1,5 & 3 & 1,0 & \\
\hline Encaminhamento Intensivo & 1 & 0,4 & 0 & 0,0 & \\
\hline Outras drogas & & & & & 0,304 \\
\hline Nenhuma Intervenção & 253 & 97,7 & 295 & 99,0 & \\
\hline Intervenção Breve & 5 & 1,9 & 3 & 1,0 & \\
\hline Encaminhamento Intensivo & 1 & 0,4 & 0 & 0,0 & \\
\hline
\end{tabular}

*p do teste de qui quadrado

\section{Discussão}

A realização deste diagnóstico no contexto escolar é imprescindível para organização de ações de promoção da saúde mental no que tange principalmente ao incentivo a comportamentos saudáveis. As características sociodemográficas dos estudantes de ensino médio em outros estudos são bem similares aos resultados encontrados em nosso cenário(4,20).

Uma pesquisa envolvendo 1.009 alunos do ensino fundamental e médio em 20 escolas públicas de Aracaju, Sergipe apresentou características sociodemográficas semelhantes, em que $56,2 \%$ eram do sexo feminino e $41,5 \%$ masculino, e a maioria estava entre 15 a 17 anos de idade ${ }^{(20)}$. Em outro estudo realizado em São Paulo, 
a idade dos alunos variou entre 14 a 18 anos, sendo a média de 14,8 anos $^{(21)}$.

Em relação às substâncias já experimentadas pelos estudantes da escola $X$ e escola $Y$, o álcool aparece em maior proporção. Este resultado tem consonância com os encontrados em outra pesquisa, que identificou consumo abusivo de álcool em 13,7\% da população brasileira, considerada alta pelos autores ${ }^{(22)}$.

No estudo com estudantes de escolas públicas e particulares, quanto ao consumo pelo menos uma vez na vida, $91 \%$ afirmaram já ter experimentado bebida alcóolica(21). Autores afirmam que a família e a cultura contribuem para o consumo precoce do álcool, uma vez que o consumo de bebidas alcoólicas é um costume pertencente à maioria das culturas, e está atrelado a festividades, tradições e cerimônias religiosas(23).

Ademais, ressalta-se que o consumo de drogas na adolescência é considerado um problema complexo, em que devem ser considerados os aspectos individuais, familiares e social, sobretudo com ênfase nos grupos de convivência que influenciam na aquisição e manutenção deste e de outros comportamentos de risco(24).

Nesse sentido, destaca-se que o Brasil é um país onde a violência mais compromete a vida da população mais jovem da sociedade. A primeira causa de morte na faixa etária dos 12 aos 29 anos é devido a causas externas, representadas pelos homicídios e acidentes de trânsito. Em ambas as situações, o uso/abuso de álcool e de outras drogas tem papel destacado na dinâmica destas causas ${ }^{(25)}$.

Nesse ínterim, evidenciou-se que além do álcool os estudantes das duas escolas investigadas também já experimentaram outras substâncias psicoativas, em que a maconha, tabaco e hipnóticos ou sedativos tiveram destaque entre as já experimentadas. Em estudo realizado em Alagoas, as cinco substâncias mais utilizadas na modalidade "uso na vida" foram: álcool, com 79,6\%; energéticos, com $25,6 \%$; cigarro, com $24,6 \%$; solventes, com 14,7\%; e ansiolíticos, com 10,1\%(7).

A necessidade de intervenção breve tanto na escola $X$ como na escola $Y$ sugere a magnitude e importância das ações de prevenção do uso abusivo de drogas. Estes resultados fornecem subsídios que auxiliam na construção de estratégias mais assertivas de intervenção no que diz respeito ao abuso e à dependência de drogas entre os jovens de escolas de ensino médio. Os resultados permitiram ainda identificar quais substâncias estão sendo consumidas e os seus níveis de necessidade de intervenção no contexto escolar.

Em estudo sobre representações sociais de professores sobre o uso abusivo de álcool e outras drogas na adolescência, percebeu-se que os educadores apresentavam representações negativas e estigmatizantes sobre o tema prevenção ao consumo de drogas no ambiente escolar (26). Autores apontam que as representações sociais sobre o uso abusivo de drogas estão inseridos em uma teia de significações sobre drogas e adolescência que podem estar relacionadas a poucas iniciativas de prevenção sobre a temática no contexto escolar. Em outro estudo também houve predominância de crenças negativas e estigmatizantes e atribuição do uso de substâncias psicoativas ao modelo moralizante(14).

O tema prevenção de uso/abuso de drogas no contexto escolar apresenta várias facetas, a principal está relacionada diretamente com a dificuldade que os professores possuem em discutir este tema de forma aprofundada. Neste contexto, enfatiza-se que para se pensar na promoção de saúde no ambiente escolar, é necessário perceber a educação para além de um processo de socialização e integração somente, mas concomitante a um caminho a partir do qual se constroem sistemas normativos e valores para a vida, marcando assim sua dimensão política(13). O trabalho de prevenção deve estar vinculado a uma proposta abrangente, em que o uso de drogas possa ser discutido em um contexto mais amplo de saúde.

Logo, tem-se a educação afetiva com ênfase na personalidade do aluno como principal método para utilizar na abordagem educativa para fins de prevenção do uso/ abuso de drogas na escola. A educação afetiva defende a modificação de fatores pessoais que são vistos como riscos ao uso de drogas, explorando situações-limite. Em primeiro lugar, deve-se priorizar o autoconhecimento, a autoestima, a autoafirmação, as relações interpessoais, a capacidade de lidar com ansiedade, a habilidade de decidir e de lidar com grupos, a capacidade de resistir às pressões grupais e a comunicação verbal ${ }^{(27)}$. Também é importante fortalecer a resiliência, o saber dizer não, a solidariedade, o pertencimento, o saber ouvir, a autonomia, a criatividade, o respeito às diferenças e aos valores. $E$, quando necessário, enfraquecer a ansiedade, o desamparo, a vulnerabilidade, a insegurança, os estigmas e preconceitos.

Nesse sentido, destaca-se o Estatuto da Criança e Adolescente e a Política Nacional de atenção integral ao usuário de álcool e outras drogas com orientações que objetivam o fortalecimento do trabalho em rede, intersetorialidade, garantia de acesso e participação do usuário no tratamento, com algumas especificidades para o adolescente, na prevenção de propagandas, comercialização e acessibilidade a essa população, vista como mais vulnerável para o uso, bem como garantir os direitos para uma vida saudável e prevenção ao consumo de drogas, com apoio de instituições públicas e privadas(28).

\section{Conclusão}

Os resultados evidenciados contribuem para compreensão do fenômeno no que diz respeito às substâncias mais consumidas, padrões de uso nocivo ou 
dependência, bem como para ampliar as possibilidades de planejar programas de prevenção primária e secundária no contexto escolar.

Portanto, os resultados permitiram conhecer as principais substâncias experimentadas pelos estudantes, sinalizando os níveis de intervenção de acordo com os propostos pelo ASSIST - teste de triagem do envolvimento com álcool, cigarro e outras substâncias. Agora as duas escolas encontram-se diante de um novo desafio e, nesta circunstância, educar para prevenção apresenta-se como a melhor alternativa para o enfrentamento do consumo de drogas entre estudantes.

Além disso, destaca-se que o estudo possibilitou expandir os conhecimentos e documentar acerca do uso de drogas no contexto escolar, que provoca a necessidade de implementar ações contínuas de prevenção ao uso de drogas e os seus agravos nesse âmbito, uma vez que se observa ser a faixa etária mais susceptível para iniciar esse consumo, demandando, portanto, políticas públicas intersetoriais que envolvam assistência social, educação e sobretudo, saúde, com estratégias efetivas, visto ser um tema complexo e plurifacetado.

No entanto, este estudo teve limitações que merecem ser pontuadas, pois, quando se trata de verificar o consumo de substâncias psicoativas dentro de instituições de ensino, estudantes que tenham envolvimento mais grave com drogas podem não frequentar mais as salas de aula ou faltar sistematicamente, não sendo captados pelo estudo. Outra situação é a veracidade das respostas, pois alguns estudantes podem ter negado o uso/consumo mesmo tendo sido ressaltado e garantido o seu anonimato.

\section{Referências}

1. Venturi G. Consumo de drogas, opinião pública e moralidade: motivações e argumentos baseados em uso. Tempo Soc. 2017; 29 (2): 159-86. doi: http://dx.doi. org/10.11606/0103-2070.ts.2017.126682.

2. United Nations Office on Drugs and Crime (UNODC). World Drug Report [Internet]. New York: UNODC Research; 2015 [cited 2018 Oct 12]. Available from: https://www.unodc.org/documents/wdr2015/World_ Drug_Report_2015.pdf

3. Nardi FL, Cunha SM, Bizarro L, Dell'Aglio DD. Drug use and antisocial behavior among adolescents attending public schools in Brazil. Trends Psychiatry Psychother. [Internet]. 2012 [cited 2018 Oct 12]; 34 (2): 80-6. Available from: http://www.scielo.br/scielo.php?script=sci_ arttext\&pid=S2237-60892012000200006\&Ing=en doi: http://dx.doi.org/10.1590/S2237-60892012000200006.

4. Raposo JCS, Costa ACQ, Valença PAM, Zarzar PM, Diniz AS, Colares $V$, et al. Uso de drogas ilícitas e binge drinking entre estudantes adolescentes. Rev Saúde Pública. [Internet]. 2017 [Acesso 14 out. 2019];
51(83): 1-7. Disponível em: http://www.revistas.usp. br/rsp/article/view/138338/133820 doi: http://dx.doi. org/10.11606/s1518-8787.2017051006863.

5. Marques ACPR, Cruz MS. O adolescente e o uso de drogas. Rev Bras Psiquiatr. 2000; 22 (supl. 2): 32-6.doi: http://dx.doi.org/10.1590/S1516-44462000000600009. 6. Sodelli M. Vulnerabilidade, Resiliência e Redes Sociais: Uso, Abuso e Dependência de Drogas. São Paulo: Red Publicações; 2015.

7. Lopes AP, Rezende MM. Consumo de substâncias psicoativas em estudantes do ensino médio. Psicol Teor Prat. [Internet]. 2014 [Acesso 14 out 2019]; 16 (2): 29-40. Disponível em: http://pepsic.bvsalud.org/scielo.php?script= sci_arttext\&pid $=$ S1516-36872014000200003\&Ing = pt\&tlng $=$ pt.

8. Malta DC, Mascarenhas MDM, Porto DL, Duarte EA, Sardinha LM, Barreto SM, et al. Prevalência do consumo de álcool e drogas entre adolescentes: análise dos dados da Pesquisa Nacional de Saúde Escolar. Rev Bras Epidemiol. 2011; 14(1): 136-46. doi: http://dx.doi. org/10.1590/S1415-790X2011000500014.

9. Oliveira EN, Olímpio ACS, Costa JBC, Moreira RMM, Oliveira LS, Silva RWS. Crack consumption: characteristics of users undergoing treatment at a Psychosocial AlcoholDrug Attention Center. SMAD. Rev Eletrônica Saúde Mental Álcool Drog. 2019; 15(4):1-8. doi: https://dx.doi. org/10.11606/issn.1806-6976.smad.2019.152138

10. Dalpiaz AK, Jacob MHVM, Silva KD, Bolson MP, Hirdes A. Fatores associados ao uso de drogas: depoimentos de usuários de um CAPS AD. Aletheia [Internet]. 2014 [Acesso 14 out 2019]; 45: 56-71. Disponível em: http:// pepsic.bvsalud.org/scielo.php?script=sci_arttext\&pid=S $141303942014000200005 \&$ Ing $=p t$

11. Cardoso LRD, Malbergier A. A influência dos amigos no consumo de drogas entre adolescentes. Estud Psicol. (Campinas). 2014; 31 (1): 65-74. doi: http://dx.doi.org/ 10.1590/0103166X2014000100007.

12. Silva AG, Rodrigues TCL, Gomes KV. Adolescência, vulnerabilidade e uso abusivo de drogas: a redução de danos como estratégia de prevenção. Rev Psicol Polít. [Internet]. 2015 [Acesso 14 out 2019]; 15 (33): 335-54. Disponível em: http://pepsic.bvsalud.org/scielo.php?script=sci_arttex t\&pid=S1519549X2015000200007\&lng=pt\&nrm=iso.

13. Moreira A, Vovio CL, Micheli D. Prevenção ao consumo abusivo de drogas na escola: desafios e possibilidades para a atuação do educador. Educ Pesqui. 2015; 41 (1): 119-35. doi: http://dx.doi.org/10.1590/S1517-97022015011670. 14. Tavares MLO, Reinaldo, MAS, Silveira BV, Pereira MO. Crenças e atitudes de educadores da rede pública de um município mineiro sobre o uso de Substâncias Psicoativas. ABCS Health Sci. 2019; 44(1):47-51. doi: https://doi.org/10.7322/abcshs.v44i1.1166

15. O Povo. Pesquisa: 32 municípios do Ceará estão com alto consumo de crack [Internet]. Jornal O Povo. Set. 2017 
[Acesso 20 out 2019]. Disponível em: https://www.opovo. com.br/noticias/fortaleza/2017/09/pesquisa-32-municipiosdo-ceara-estao-com-alto-consumo-de-crack.html.

16. Carminatti VP. Validação concorrente e confiabilidade da versão brasileira do ASSIST-WHO (Alcohol Smoking and Substance Involvement Screening Test) para adolescentes 'Dissertação]. São Paulo (SP): Universidade Federal de São Paulo; 2010.

17. Henrique IFS, De Micheli D, Lacerda RB, Lacerda LA, Formigoni MLOS. Validação da versão brasileira do teste de triagem do envolvimento com álcool, cigarro e outras substâncias (ASSIST). Rev Assoc Med Bras. 2014; 50(2): 199-206. doi: http://dx.doi.org/10.1590/ S0104-42302004000200039.

18. Humeniuk R, Henry-Edwards S, Ali R, Poznyak V, Monteiro MG. The Alcohol, Smoking and Substance Involvement Screening Test (ASSIST): manual for use in primary care [Internet]. Genebra $(\mathrm{CH})$ : World Health Organization; 2010 [cited 2019 Oct 21]. Available from: https://apps.who.int/iris/handle/10665/44320.

19. Ministério da Saúde (BR). Conselho Nacional de Saúde. Resolução n.466, de 12 de dezembro de 2012 [Internet]. Aprova diretrizes e normas regulamentadoras de pesquisas envolvendo seres humanos. Brasília: Diário Oficial da União; 2012 [Acesso 21 out 2019]. Disponível em: http://conselho.saude.gov.br/resolucoes/2012/ Reso466.pdf.

20. Andrade ME, Santos IHF, Souza AAM, Silva ACS, Leite TS, Oliveira CCC, et al. Experimentação de substâncias psicoativas por estudantes de escolas públicas. Rev Saúde Pública. 2017; 51 (82): 1-9. doi: http://dx.doi. org/10.11606/s1518-8787.2017051006929.

21. Oliveira HF, Martins LC, Reato LFN, Akerman M. Fatores de risco para uso do tabaco em adolescentes de duas escolas do município de Santo André, São Paulo. Rev Paul Pediatr. 2010; 28 (2): 200-7. doi: http://dx.doi. org/10.1590/S0103 05822010000200012.

22. Garcia LP, Freitas LRS. Consumo abusivo de álcool no Brasil: resultados da Pesquisa Nacional de Saúde 2013. Epidemiol Serv Saúde. 2015;24 (2): 227-37. doi: http://dx.doi.org/10.5123/S1679-49742015000200005. 23. Rodrigues G, Krindges CA. Consequências psicossociais atreladas ao consumo precoce de bebida alcoólica. Rev Psicol IMED. 2017; 9(2): 61-76. doi: http://dx.doi.org/10.18256/2175-5027.2017.v9i2.2087.
24. Zappe JG, Dapper F. Drogadição na Adolescência: Família como Fator de Risco ou Proteção. Rev Psicol IMED. 2017; 9 (1): 140-58. doi: http://dx.doi.org/10.18256/ 2175-5027.2017.v9i1.1616.

25. Lima JMB. Alcoologia: o alcoolismo na perspectiva da saúde pública. Rio de Janeiro: MEDBOOK; 2008.

26. Araldi JC, Njaine K, Oliveira MC, Ghizoni AC. Representações sociais de professores sobre o uso abusivo de álcool e outras drogas na adolescência: repercussões nas ações de prevenção na escola. Interface. $2012 ; 16(40)$ : 135-48. doi: http://dx.doi. org/10.1590/S1414-32832012005000002.

27. Fonseca MS. Como prevenir o abuso de drogas nas escolas? Psicol Esc Educ.. (Campinas) 2006; 10 (2): 339-41. doi: http://dx.doi.org/10.1590/S1413-85572006000200018. 28. Decreto n 9761 (BR), de 11 de abril de 2019. Aprova a Política Nacional sobre Drogas. Diário Oficial da União. 11 abr 2019.

\section{Contribuição dos autores}

Concepção e desenho da pesquisa: Eliany Nazaré Oliveira, Joyce Mazza Nunes, Maristela Inês Osawa Vasconcelos e Mariana Rodrigues Bezerra. Obtenção de dados: Eliany Nazaré Oliveira, Joyce Mazza Nunes, Lorenna Saraiva Viana e Mariana Rodrigues Bezerra. Análise e interpretação dos dados: Eliany Nazaré Oliveira, Joyce Mazza Nunes, Maristela Inês Osawa Vasconcelos, Roberta Magda Martins Moreira e Mariana Rodrigues Bezerra. Redação do manuscrito: Lorenna Saraiva Viana, Roberta Magda Martins Moreira e Mariana Rodrigues Bezerra. Revisão crítica do manuscrito quanto ao conteúdo intelectual importante: Eliany Nazaré Oliveira, Joyce Mazza Nunes, Maristela Inês Osawa Vasconcelos, Lorenna Saraiva Viana e Roberta Magda Martins Moreira.

Todos os autores aprovaram a versão final do texto.

Conflito de interesse: os autores declararam que não há conflito de interesse. 